\title{
Computing Controllers for Nonlinear Hybrid Systems ${ }^{\star}$
}

\author{
Claire Tomlin ${ }^{1}$, John Lygeros ${ }^{2}$, and Shankar Sastry ${ }^{2}$ \\ 1 Department of Aeronautics and Astronautics \\ Stanford University, Stanford CA 94305-4035 \\ tomlin@leland.stanford.edu \\ 2 Department of Electrical Engineering and Computer Sciences \\ University of California, Berkeley CA 94720-1770 \\ \{lygeros, sastry\}@eecs . berkeley.edu
}

\begin{abstract}
We discuss a procedure for synthesizing controllers for safety specifications for hybrid systems. The procedure depends on the construction of the set of states of a continuous dynamical system that can be driven to a subset of the state space, avoiding another subset of the state space (the Reach-Avoid set). We present a new characterization of the Reach-Avoid set in terms of the solution of a pair of coupled Hamilton-Jacobi partial differential equations. We also discuss a computational algorithm for solving such partial differential equations and demonstrate its effectiveness on numerical examples.
\end{abstract}

\section{Introduction}

The synthesis of controllers that meet safety specifications for discrete, continuous and hybrid systems has attracted considerable attention (see I $>34$ for an overview). Our work has been based on casting the problem as a two player, zero sum game, between a controller, that tries to ensure that the safety specification is satisfied and a disturbance (that includes the nondeterminism of the system), that tries to violate the safety specification 5 . In 6 we proposed a procedure for systematically carrying out the controller synthesis for general hybrid systems. The procedure relies on the solution of partial differential equations (PDEs) 7 , known as the Hamilton-Jacobi equations. Here, we bring the synthesis procedure one step closer to implementation, by proposing a numerical scheme for solving these partial differential equations.

In Section $\square$ we briefly review the modeling formalism and the controller synthesis problem introduced in 5 . In Section 3 we review the algorithm proposed in 6 for solving the controller synthesis problem. The algorithm requires the computation of the set of states of a continuous dynamical system that can be driven to a given subset of the state space, avoiding another subset of the state

\footnotetext{
* Research supported by NASA under grant NAG 2-1039, by ONR under grant N00014-97-1-0946, by DARPA under contract F33615-98-C-3614 and by a Zonta Postgraduate Fellowship.
} 
space (the Reach-Avoid set). We introduce a new procedure for characterizing the Reach-Avoid set, in terms of the solution to a pair of coupled HamiltonJacobi PDEs. The advantage of this new characterization (over the single PDE characterization of 7 , for example) is that it can deal with situations where the closures of the Reach and Avoid sets overlap, without resorting to approximation. It also remains closer to the Hamilton-Jacobi PDE arising in purely continuous pursuit evasion problems, which makes it easier to carry classical results over to the hybrid domain.

An analytical solution to the Hamilton Jacobi PDEs is likely to be impossible to obtain for most realistic examples. For the class of systems we consider the situation is additionally complicated by the fact that the initial conditions of the PDE may be non-smooth; shocks, or discontinuities in the spatial variable as the temporal variable evolves, may develop; the right hand side of the PDE may be non-smooth, due to the optimal (typically bang-bang) controls; the right hand side of the PDE may be discontinuous, due to saturation effects introduced to guarantee the monotonicity of the Reach-Avoid set. In Section $\square$ we present a procedure for numerically computing the Reach-Avoid set, based on the level set method of $x$. The advantage of this method is that it can systematically deal with all the technical problems highlighted above, based on the viscosity solution concept for the PDEs. A brief comparison with other techniques proposed in the literature for computing or approximating the reach set of hybrid systems is also given. We demonstrate the application of this approach to a new example from aircraft collision avoidance, developed from 9 (Section 5 .

\section{Model}

For a finite collection $V$ of variables, let $\mathbf{V}$ denote the set of valuations of these variables, i.e. the set of all possible assignments of the variables in $V$. For example, if $x$ is a state variable taking values in $\mathbb{R}^{n}$ we write $X=\{x\}$ with $\mathbf{X}=\mathbb{R}^{n}$. By abuse of notation, we use lower case letters to denote both a variable and its valuation; the interpretation should be clear from the context. We call a variable discrete if its set of valuations is countable and continuous if it is a subset of Euclidean space. We assume the discrete topology for countable sets and the Euclidean metric topology for subsets of Euclidean space. For a topological space $X$ and a set $K \subseteq X$ we denote by $K^{c}$ the complement, by $\bar{K}$ the closure, by $K^{o}$ the interior, and by $\partial K=\bar{K} \backslash K^{\circ}$ the boundary of $K$ in the topology of $X$. Given a set of valuations $W \subseteq \mathbf{V}$ and a subset of the variables $V^{\prime} \subset V$ we denote by $\left.W\right|_{V^{\prime}} \subset \mathbf{V}^{\prime}$ the restriction of $W$ to $V^{\prime}$.

\subsection{Hybrid Automata}

Definition $1 A$ hybrid automaton, $H$, is a collection $(X, V, I, f, E, \phi)$, with:

- State and input variables: $X$ and $V$ are disjoint collections of state and input variables. We assume that $X=X_{D} \cup X_{C}$ and $V=V_{D} \cup V_{C}$, where $X_{C}$ 
and $V_{C}$ contain continuous, and $X_{D}$ and $V_{D}$ discrete variables. We refer to the valuations $x \in \mathbf{X}$ and $v \in \mathbf{V}$ as the state and the input of the hybrid automaton.

- Initial states: $I \subset \mathbf{X}$ is a set of initial valuations of the state variables.

- Continuous evolution: $f: \mathbf{X} \times \mathbf{V} \rightarrow T \mathbf{X}_{\mathbf{C}}$ is a vector field.

- Discrete transitions: $E \subset \mathbf{X} \times \mathbf{V} \times \mathbf{X}$ is a set of discrete transitions.

- Admissible inputs: $\phi: \mathbf{X} \rightarrow 2^{\mathbf{V}}$ gives the set of admissible inputs at a given state $x \in \mathbf{X}$.

To fix notation we let $\mathbf{X}_{\mathbf{C}} \subseteq \mathbb{R}^{n}$ and $\mathbf{V}_{\mathbf{C}} \subseteq \mathbb{R}^{m}$. For technical conditions imposed to ensure well posedness see 5 .

Definition $2 A$ hybrid time trajectory, $\tau$, is a finite or infinite sequence of intervals $\tau=\left\{I_{i}\right\}$ of the real line, starting with $I_{0}$ and satisfying:

- $I_{i}$ is closed unless $\tau$ is a finite sequence and $I_{i}$ is the last interval, in which case it is left closed but can be right open.

- Let $I_{i}=\left[\tau_{i}, \tau_{i}^{\prime}\right]$. Then for all $i \tau_{i} \leq \tau_{i}^{\prime}$ and for $i>0, \tau_{i}=\tau_{i-1}^{\prime}$.

We denote by $\mathcal{T}$ the set of all hybrid time trajectories.

Definition 3 An execution of a hybrid automaton $H$ is a collection $(\tau, x, v)$ with $\tau \in \mathcal{T}, x: \tau \rightarrow \mathbf{X}$, and $v: \tau \rightarrow \mathbf{V}$ which satisfies:

- Initial Condition: $x\left(\tau_{0}\right) \in I$.

- Discrete Evolution: $\left(x\left(\tau_{i-1}^{\prime}\right), v\left(\tau_{i-1}^{\prime}\right), x\left(\tau_{i}\right)\right) \in E$, for all $i$.

- Continuous Evolution: for all $i$ with $\tau_{i}<\tau_{i}^{\prime}, x$ is continuous and $v$ is piecewise continuous in $\left[\tau_{i}, \tau_{i}^{\prime}\right]$ and for all $t \in\left[\tau_{i}, \tau_{i}^{\prime}\right),(x(t), v(t), x(t)) \in E$. Moreover, for all $t \in\left[\tau_{i}, \tau_{i}^{\prime}\right]$ where $v$ is continuous $\frac{d}{d t}\left(\left.x(t)\right|_{X_{C}}\right)=f(x(t), v(t))$.

- Input Constraints: for all $t \in \tau, v(t) \in \phi(x(t))$.

We use $\chi$ to denote an execution of $H$ and $\mathcal{H}$ to denote the set of all executions of $H$. We use $x^{0}=x\left(\tau_{0}\right)$ to denote the initial state of an execution.

A property, $P$, of a hybrid automaton $H$ is a map:

$$
P: \mathcal{H} \rightarrow\{\text { True, False }\}
$$

We say an execution $\chi \in \mathcal{H}$ satisfies property $P$ if $P(\chi)=$ True; we say a hybrid automaton satisfies a property $P$ if $P(\chi)=$ True for all $\chi \in \mathcal{H}$. Given a set $F \subset \mathbf{X}$ we define a safety property, denoted by $\square F$, by:

$$
\square F(\chi)=\left\{\begin{array}{l}
\text { True if } \forall t \in \tau, x(t) \in F \\
\text { False otherwise }
\end{array}\right.
$$




\subsection{Controller Synthesis}

Assume that we are given a hybrid automaton $H$, which we refer to as the plant, and we are asked to control it using its input variables so that its executions satisfy certain properties. For the purposes of control the input variables of the plant are partitioned into two classes: controls and disturbances. We write $V=U \cup D$ where $U$ and $D$ are respectively control and disturbance variables. The interpretation is that the controls can be influenced using a controller, in an attempt to guide the system, whereas the disturbances are determined by the environment and may potentially disrupt the controller's plans.

An instance of the controller synthesis problem consists of a pair, $(H, P)$, of a plant hybrid automaton and a property of that automaton. In this paper we restrict our attention to controller synthesis problems where $P=\square F$. A static state feedback controller for $H$ is a map:

$$
g: \mathbf{X} \rightarrow 2^{\mathbf{U}}
$$

Given a plant automaton $H$ and a controller $g$ for $H$ one can define the set of closed loop executions as:

$$
\mathcal{H}_{g}=\{(\tau, x,(u, d)) \in \mathcal{H} \mid \forall t \in \tau \quad u(t) \in g(x(t))\}
$$

It is easy to see that this is precisely the set of executions of another hybrid automaton, $H_{g}$. We say that controller $g$ solves the synthesis problem $(H, \square F)$ if $H_{g}$ satisfies $\square F$. It can be shown 5 that for controller synthesis problems of the form $(H, \square F)$, one can restrict attention to memoryless controllers without loss of generality.

A subset $W \subseteq \mathbf{X}$ is controlled invariant if the controller synthesis problem $(H, \square W)$ can be solved when $I=W$. It can be shown 5 that the controller synthesis problem $(H, \square F)$ can be solved if and only if there exists a unique maximal controlled invariant subset of $F$. In the next sections we highlight a procedure (introduced in 6 ) for computing this subset.

\section{Controller Synthesis for Hybrid Systems}

\subsection{Construction of Controlled Invariant Sets}

For the synthesis problem $(H, \square F)$ we seek to construct the largest set of states for which the control $u$ can guarantee that the property $\square F$ is satisfied, despite the action of the disturbance $d$. We first introduce some notation. For any $v=$ $(u, d)$ define the set:

$$
\operatorname{Inv}(v)=\{x \in \mathbf{X} \mid v \in \phi(x) \text { and }(x, v, x) \in E\}
$$

For a state $x \in \mathbf{X}$ and input $v=(u, d)$ consider the sets:

$$
\operatorname{Next}(x, v)= \begin{cases}\left\{x^{\prime} \in \mathbf{X} \mid\left(x, v, x^{\prime}\right) \in E\right\} & \text { if } v \in \phi(x) \\ \emptyset & \text { if } v \notin \phi(x)\end{cases}
$$


$\operatorname{Inv}(v)$ is the set of states from which continuous evolution is possible under input $v$, while $\operatorname{Next}(x, v)$ is the set of states that can be reached from state $x$ under input $v$ through a discrete transition. Abusing notation slightly, for any set $K \subseteq \mathbf{X}$ and input $v=(u, d)$ we define the successor of $K$ under $v$ as the set:

$$
\operatorname{Next}(K, v)=\bigcup_{x \in K} \operatorname{Next}(x, v)
$$

For any set $K \subseteq \mathbf{X}$ we define the controllable predecessor of $K, \operatorname{Pre}_{u}(K)$, and the uncontrollable predecessor of $K, \operatorname{Pre}_{d}(K)$, by:

$$
\begin{aligned}
& \operatorname{Pre}_{u}(K)=\{x \in \mathbf{X} \mid \exists u \in \mathbf{U} \forall d \in \mathbf{D} x \notin \operatorname{Inv}(v) \text { and } N \operatorname{ext}(K,(u, d)) \subseteq K\} \cap K \\
& \operatorname{Pre}_{d}(K)=\left\{x \in \mathbf{X} \mid \forall u \in \mathbf{U} \exists d \in \mathbf{D} \operatorname{Next}(K,(u, d)) \cap K^{c} \neq \emptyset\right\} \cup K^{c}
\end{aligned}
$$

$\operatorname{Pre}_{u}(K)$ contains all states in $K$ for which $u$ can force a transition back into $K$. $\operatorname{Pre}_{d}(K)$, on the other hand, contains all states outside $K$, as well as all states from which a transition outside of $K$ is possible whatever $u$ does. It is easy to show that:

Proposition $1 \operatorname{Pre}_{u}(K) \cap \operatorname{Pre}_{d}(K)=\emptyset$.

The controllable and uncontrollable predecessors will be used in the discrete part of the algorithm for determining controlled invariant subsets. For the continuous part we introduce the Reach-Avoid operator:

Definition 4 (Reach-Avoid) For two disjoint sets $B \subseteq \mathbf{X}$ and $G \subseteq \mathbf{X}$, define the Reach-Avoid operator as:

$$
\begin{aligned}
\operatorname{Reach}(B, G)= & \left\{x^{0} \in \mathbf{X} \mid \forall u \in \mathcal{U}_{f} \exists d \in \mathcal{D} \text { and } t \geq 0\right. \text { such that } \\
& x(t) \in B \text { and } x(s) \notin G \text { for all } s \in[0, t]\}
\end{aligned}
$$

Here $\mathcal{U}_{f}$ denotes the set of all $\mathbf{U}$-valued feedback strategies, $\mathcal{D}$ denotes the set of piecewise continuous functions from the real line to $\mathbf{D}$ and $x(\cdot)$ the (unique) continuous state trajectory starting at $x(0)=x^{0}$ under input $(u, d)$.

The set $\operatorname{Reach}(B, G)$ contains the states from which, for all $u(\cdot)$, there exists a $d(\cdot)$, such that the state trajectory can be driven to $B$ while avoiding an "escape" set $G$.

Consider the following algorithm.

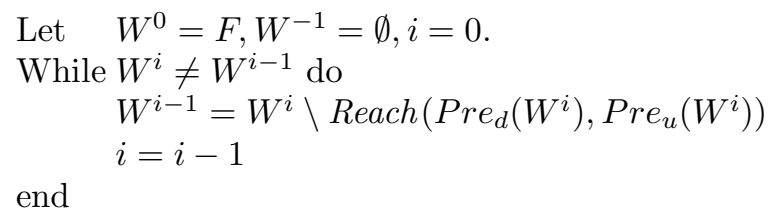

In the first step of this algorithm, we remove from $F$ all states for which there is a disturbance $d(\cdot)$ which through continuous evolution can bring the system either outside $F$, or to states from which a transition outside $F$ is possible, without first 
touching the set of states from which a transition keeping the system inside $F$ can be forced. Since at each step $W^{i-1} \subseteq W^{i}$, the set $W^{i}$ decreases monotonically as $i$ decreases. If the algorithm terminates, we denote the fixed point by $W^{*}$. In this case, $W^{*}$ can be shown to be the largest controlled invariant subset contained in $F$.

\subsection{Characterization of the Reach-Avoid Set}

To implement this algorithm, we need to calculate Pre $_{u}$, Pre $_{d}$, and Reach. The calculation of $\mathrm{Pre}_{u}$ and $\mathrm{Pre}_{d}$ is conceptually straight forward and can be done by inverting the transition relation $E$. In this section we discuss the computation of the Reach set. By definition, the discrete state remains constant along continuous evolution. Therefore, Reach $\left(\operatorname{Pre}_{d}\left(W^{i}\right), \operatorname{Pre}_{u}\left(W^{i}\right)\right)$ can be computed in "parallel" for each discrete state (see 10 for a similar partition proposed for optimal control problems). In the following analysis, we describe this calculation for one discrete state $q \in \mathbf{X}_{\mathbf{D}}$. Abusing notation, we use from now on $x$ to denote the continuous part of the state, with the discrete part frozen at $q$.

For two disjoint sets $B \subseteq \mathbf{X}_{\mathbf{C}}$ and $G \subseteq \mathbf{X}_{\mathbf{C}}$, assume that there exist differentiable functions $l_{B}: \mathbf{X}_{\mathbf{C}} \rightarrow \mathbb{R}$ and $l_{G}: \mathbf{X} \rightarrow \mathbb{R}$ such that $B \triangleq\left\{x \in \mathbf{X}_{\mathbf{C}} \mid l_{B}(x) \leq\right.$ $0\}$ and $G \triangleq\left\{x \in \mathbf{X}_{\mathbf{C}} \mid l_{G}(x) \leq 0\right\} \|$. Consider the following system of coupled Hamilton-Jacobi equations:

$$
-\frac{\partial J_{B}(x, t)}{\partial t}= \begin{cases}H_{B}^{*}\left(x, \frac{\partial J_{B}(x, t)}{\partial x}\right) & \text { for }\left\{x \in X \mid J_{B}(x, t)>0\right\} \\ \min \left\{0, H_{B}^{*}\left(x, \frac{\partial J_{B}(x, t)}{\partial x}\right)\right\} & \text { for }\left\{x \in X \mid J_{B}(x, t) \leq 0\right\}\end{cases}
$$

and

$$
-\frac{\partial J_{G}(x, t)}{\partial t}= \begin{cases}H_{G}^{*}\left(x, \frac{\partial J_{G}(x, t)}{\partial x}\right) & \text { for }\left\{x \in X \mid J_{G}(x, t)>0\right\} \\ \min \left\{0, H_{G}^{*}\left(x, \frac{\partial J_{G}(x, t)}{\partial x}\right)\right\} & \text { for }\left\{x \in X \mid J_{G}(x, t) \leq 0\right\}\end{cases}
$$

where $J_{B}(x, 0)=l_{B}(x)$ and $J_{G}(x, 0)=l_{G}(x)$, and

$$
\begin{aligned}
& H_{B}^{*}\left(x, \frac{\partial J_{B}}{\partial x}\right)=\left\{\begin{array}{l}
0, \text { for }\left\{x \in X \mid J_{G}(x, t) \leq 0\right\} \\
\max _{u \in \mathbf{U}} \min _{d \in \mathbf{D}} \frac{\partial J_{B}}{\partial x} f(x, u, d), \text { otherwise }
\end{array}\right. \\
& H_{G}^{*}\left(x, \frac{\partial J_{G}}{\partial x}\right)=\left\{\begin{array}{l}
0, \text { for }\left\{x \in X \mid J_{B}(x, t) \leq 0\right\} \\
\min _{u \in \mathbf{U}} \max _{d \in \mathbf{D}} \frac{\partial J_{G}}{\partial x} f(x, u, d), \text { otherwise }
\end{array}\right.
\end{aligned}
$$

Equation (II) describes the evolution of the set $B$ under the Hamiltonian $H_{B}^{*}$. This is the solution to the " $\max _{u} \min _{d}$ " game for reachability in purely continuous systems (see for example $b$ ), with the modification that $H_{B}^{*}=0$ in $\left\{x \in \mathbf{X}_{\mathbf{C}} \mid J_{G}(x, t) \leq 0\right\}$. This ensures that the evolution of $J_{B}(x, t)$ is frozen once this set is reached. Similarly, equation II describes the evolution of the set $G$ under the Hamiltonian $H_{G}^{*}$. Here a " $\min _{u} \max _{d}$ " is used, since it is assumed

${ }^{1}$ More generally, $B$ and $G$ may be expressed as the maximum of a set of differentiable functions, as discussed in Section $y$ 


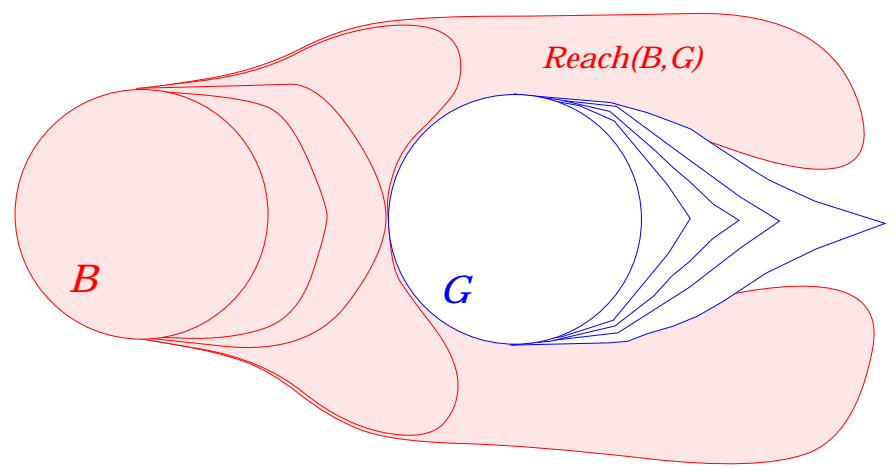

Fig. 1. The computation of $\operatorname{Reach}(B, G)$ in a single discrete state $q$.

that the control tries to push the system into $G$, to escape from $B$. $H_{G}^{*}=0$ in $\left\{x \in \mathbf{X}_{\mathbf{C}} \mid J_{B}(x, t) \leq 0\right\}$ to ensure that the evolution of $J_{G}(x, t)$ is frozen once this set is reached. In both cases we perform the calculation in "backwards time": we start the calculation at a fixed final time $t=0$ and evolve backwards to a free initial time $t<0$.

Note that in both games, the disturbance is given the advantage by assuming that the control plays first. In the following sequence of Lemmas, we prove that the resulting set $\left\{x \in \mathbf{X}_{\mathbf{C}} \mid J_{B}(x, t)<0\right\}$ contains neither $G$ nor states for which there is a control which drives the system into $G$; and the set $\{x \in$ $\left.\mathbf{X}_{\mathbf{C}} \mid J_{G}(x, t)<0\right\}$ contains neither $B$ nor states for which there is a disturbance which drives the system into $B$. We then prove that $\left\{x \in \mathbf{X}_{\mathbf{C}} \mid J_{B}(x, t)<0\right\}$ is the set $\operatorname{Reach}(B, G)$. Figure II illustrates an example.

Assume that differentiable functions $J_{B}$ and $J_{G}$ satisfying the above partial differential equations exist. For all $t \leq 0$, let

$$
B(t) \triangleq\left\{x \in \mathbf{X}_{\mathbf{C}} \mid J_{B}(x, t) \leq 0\right\} \quad G(t) \triangleq\left\{x \in \mathbf{X}_{\mathbf{C}} \mid J_{G}(x, t) \leq 0\right\}
$$

Note that $B=B(0)$ and $G=G(0)$.

Lemma 1 For all $t_{2} \leq t_{1} \leq 0, B\left(t_{1}\right) \subseteq B\left(t_{2}\right)$ and $G\left(t_{1}\right) \subseteq G\left(t_{2}\right)$.

Proof: Since $\frac{\partial J_{B}}{\partial t} \geq 0$ when $J_{B}(x, t) \leq 0$ and $\frac{\partial J_{G}}{\partial t} \geq 0$ when $J_{G}(x, t) \leq 0$, both $J_{B}(x, t)$ and $J_{G}(x, t)$ are monotone non-increasing functions of $-t$ when $J_{B}(x, t) \leq 0$ and $J_{G}(x, t) \leq 0$. Thus, as $t$ decreases, the sets $B(t)$ and $G(t)$ do not decrease.

Lemma 2 If $B^{\circ}(0) \cap G^{\circ}(0)=\emptyset$ then for all $t \leq 0, B^{\circ}(t) \cap G^{\circ}(t)=\emptyset$.

Proof: Assume, for the sake of contradiction, that $x^{0} \in B^{\circ}(t) \cap G^{\circ}(t)$ for some $t=t_{1}<0$, i.e. that

$$
J_{B}\left(x^{0}, t_{1}\right)<0 \quad \text { and } \quad J_{G}\left(x^{0}, t_{1}\right)<0
$$


We first show that $J_{B}\left(x^{0}, 0\right) \geq 0$ and $J_{G}\left(x^{0}, 0\right) \geq 0$ (i.e., $x^{0}$ is outside of both $B$ and $G$ at $t=0)$. Suppose this is not true, i.e. suppose for example that $J_{B}\left(x^{0}, 0\right)<0$ and $J_{G}\left(x^{0}, 0\right) \geq 0$. Then for all $t \leq 0$

$$
\frac{\partial J_{G}\left(x^{0}, t\right)}{\partial t}=0
$$

which implies that $J_{G}\left(x^{0}, t\right)=J_{G}\left(x^{0}, 0\right) \geq 0$ for all $t \leq 0$, which contradicts I. A similar argument holds for the case in which $J_{B}\left(x^{0}, 0\right) \geq 0$ and $J_{G}\left(x^{0}, 0\right)<0$.

Thus $J_{B}\left(x^{0}, 0\right) \geq 0$ and $J_{G}\left(x^{0}, 0\right) \geq 0$. Since $J_{B}\left(x, t_{1}\right)<0$, there exists $t_{2} \in\left[t_{1}, 0\right]$ such that $J_{B}\left(x^{0}, t_{2}\right)=0$, and for all $t \in\left[t_{1}, t_{2}\right], J_{B}\left(x^{0}, t\right) \leq 0$. Thus for at least some interval in $\left[t_{1}, t_{2}\right], J_{G}\left(x^{0}, t\right)>0$ (to allow $J_{B}\left(x^{0}, t\right)$ to decrease in this interval) and

$$
\frac{\partial J_{G}\left(x^{0}, t\right)}{\partial t}=0
$$

But this contradicts the assumption that $x^{0} \in G^{\circ}\left(t_{1}\right)$. A symmetric argument holds for $J_{G}\left(x^{0}, t\right)$.

Lemma 3 For all $t \leq 0, B(t) \cap G(t)=\partial B(t) \cap \partial G(t)$. Moreover, for all $t^{\prime} \leq t$, $B(t) \cap G(t) \subseteq \partial B\left(t^{\prime}\right) \cap \partial G\left(t^{\prime}\right)$.

\section{Proof:}

$$
B(t) \cap G(t)=\left(B^{\circ}(t) \cap G^{\circ}(t)\right) \cup(\partial B(t) \cap \partial G(t)) \cup\left(B^{\circ}(t) \cap \partial G(t)\right) \cup\left(\partial B(t) \cap G^{\circ}(t)\right)
$$

From Lemma $2\left(B^{\circ}(t) \cap G^{\circ}(t)\right)=\emptyset$.

Assume that for some $t=t_{1}<0, x^{0} \in B^{\circ}(t) \cap \partial G(t)$. Therefore, $J_{B}\left(x^{0}, t_{1}\right)<$ 0 and $J_{G}\left(x^{0}, t_{1}\right)=0$. Therefore, there exists $t_{2} \in\left[t_{1}, 0\right]$ such that $J_{B}\left(x^{0}, t_{2}\right)=0$ and for all $t \in\left[t_{1}, t_{2}\right], J_{B}\left(x^{0}, t\right) \leq 0$. Thus for some interval of $\left[t_{1}, t_{2}\right], J_{G}\left(x^{0}, t\right)>$ 0 and

$$
\frac{\partial J_{G}\left(x^{0}, t\right)}{\partial t}=0
$$

which contradicts the assumption that $x^{0} \in \partial G\left(t_{1}\right)$. Thus $B^{\circ}(t) \cap \partial G(t)=\emptyset$. A symmetric argument holds for $x^{0} \in \partial B(t) \cap G^{\circ}(t)$ for $t=t_{1}<0$, thus $\partial B(t) \cap G^{\circ}(t)=\emptyset$.

Therefore, $B(t) \cap G(t)=\partial B(t) \cap \partial G(t) . B(t) \cap G(t) \subseteq \partial B\left(t^{\prime}\right) \cap \partial G\left(t^{\prime}\right)$ for $t^{\prime} \leq t$ follows from Lemma 1

Theorem 1 (Characterization of Reach-Avoid) Assume that $J_{B}(x, t)$ (or $J_{G}(x, t)$ respectively) satisfies the Hamilton-Jacobi equation 170 (or 11 ) respectively), and that it converges uniformly in $x$ as $t \rightarrow-\infty$ to a function $J_{B}^{*}(x)$ (or $J_{G}^{*}(x)$ respectively). Then,

$$
\operatorname{Reach}(B, G)=\left\{x \in \mathbf{X}_{\mathbf{C}} \mid J_{B}^{*}(x)<0\right\}
$$


Proof: Let $x^{0} \in\left\{x \in \mathbf{X}_{\mathbf{C}} \mid J_{B}^{*}(x)<0\right\}$. Therefore, by construction, for all $u(\cdot) \in \mathcal{U}$ there exists $d(\cdot) \in \mathcal{D}$ such that the state trajectory $x(\cdot)$, starting at $x^{0}$, will eventually enter $B$. Also, by Lemma 2 $J_{G}\left(x^{0}\right)>0$. Thus $\forall u(\cdot) \in \mathcal{U}$, $\exists d(\cdot) \in \mathcal{D}$, such that the state trajectory $x(\cdot)$ starting at $x^{0}$ never enters $G$. Thus, $\left\{x \in \mathbf{X}_{\mathbf{C}} \mid J_{B}^{*}(x)<0\right\} \subseteq \operatorname{Reach}(B, G)$.

Now let $x^{0} \in\left\{x \in \mathbf{X}_{\mathbf{C}} \mid J_{B}^{*}(x) \geq 0\right\}$. Assume for the sake of contradiction that for all $u(\cdot) \in \mathcal{U}$, there exists a $d(\cdot) \in \mathcal{D}$ such that the trajectory $x(\cdot)$, starting at $x^{0}$, enters $B$. Since for all $x \in B, J_{B}^{*}(x)<0$, there exists a time $t_{1}>0$ at which this trajectory crosses $\left\{x \in \mathbf{X}_{\mathbf{C}} \mid J_{B}^{*}(x)=0\right\}$. However, for all $x$ such that $J_{B}^{*}(x)=0$, there must exist a $u \in \mathbf{U}$ such that for all $d \in \mathbf{D}, f(x, u, d)$ points outside of $\left\{x \in \mathbf{X}_{\mathbf{C}} \mid J_{B}^{*}(x)<0\right\}$. This contradicts the assumption of existence of a $d(\cdot)$ which drives the system to $B$. Thus, $\operatorname{Reach}(B, G) \subseteq\left\{x \in \mathbf{X}_{\mathbf{C}} \mid J_{B}^{*}(x)<\right.$ $0\}$.

Using the function $J_{B}^{*}$ obtained once the algorithm has converged, a controller which renders $W^{*}$ invariant can be constructed:

$$
g(x)=\left\{\begin{array}{l}
\left\{\left.u \in \phi(x)\right|_{U}|\forall d \in \phi(x)|_{D} \operatorname{Next}(x,(u, d)) \subseteq W^{*}\right\}, \text { if } x \in\left(W^{*}\right)^{o} \\
\left\{\left.u \in \phi(x)\right|_{U}|\forall d \in \phi(x)|_{D}\left(\frac{\partial J_{B}^{*}(x)}{\partial x} f(x,(u, d)) \geq 0 \wedge x \in \operatorname{Inv}(u, d)\right)\right. \\
\left.\quad \vee\left(N e x t(x,(u, d)) \subseteq W^{*} \wedge x \notin \operatorname{Inv}(u, d)\right)\right\}, \text { if } x \in \partial W^{*} \\
\left.\phi(x)\right|_{U}, \text { if } x \in\left(W^{*}\right)^{c}
\end{array}\right.
$$

Here $\wedge$ stands for the logical $A N D$ and $\vee$ for the logical $O R$.

In general, one cannot expect to solve for $W^{*}$ using a finite computation. The class of hybrid systems for which algorithms like the one presented here are guaranteed to terminate is known to be restricted 4 . Techniques have been proposed to resolve this problem, making use of approximation schemes to obtain estimates of the solution (some are discussed in the next section). In practice, we are helped by the fact that we are usually interested in finite time computations, rather than computing for $t \rightarrow-\infty$ or until a fixed point is reached.

Another problem is the requirement that the controller resulting from our algorithm be non-Zeno (does not enforce the safety requirement by preventing time from diverging). The algorithm proposed here has no way of preventing such behavior. A practical method of resolving the Zeno problem is adding a requirement that the amount of time the system remains in each discrete state is bounded below by a positive number (representing, for example, the clock period of a digital computer).

\section{Computation using Level Set Methods}

In practice, the usefulness of the algorithm for hybrid controller synthesis depends on our ability to efficiently compute the optimal control and disturbance trajectories $\left(u^{*}(\cdot), d^{*}(\cdot)\right)$, that arise from the solution of the Hamilton-Jacobi partial differential equations. Numerical solutions are potentially complicated by the fact that the right hand side of proposed PDEs is non-smooth, the initial data $F$ may have non-smooth boundary, $\left(u^{*}(\cdot), d^{*}(\cdot)\right)$ may be discontinuous, 
and the solution may develop shocks over time. New optimal control tools 11 can make the computation of $\left(u^{*}(\cdot), d^{*}(\cdot)\right)$ feasible, at least numerically. In this section, we discuss a numerical technique developed by Osher and Sethian which computes the correct viscosity solution to the proposed PDEs, ensuring that discontinuities are preserved. We then compare it briefly with other approximation techniques found in the literature.

\subsection{A Level Set Method for Boundary Approximation}

Consider the Hamilton-Jacobi equation:

$$
-\frac{\partial J(x, t)}{\partial t}= \begin{cases}H^{*}\left(x, \frac{\partial J(x, t)}{\partial x}\right) & \text { for }\left\{x \in \mathbf{X}_{\mathbf{C}} \mid J(x, t)>0\right\} \\ \min \left\{0, H^{*}\left(x, \frac{\partial J(x, t)}{\partial x}\right)\right\} & \text { for }\left\{x \in \mathbf{X}_{\mathbf{C}} \mid J(x, t) \leq 0\right\}\end{cases}
$$

with boundary condition $J(x, 0)=l(x)$. A viscosity solution $[\mathrm{I2} \mathrm{I} \cdot 3$ to 20 is the solution as $\epsilon \rightarrow 0$ to the partial differential equation:

$$
-\frac{\partial J_{\epsilon}(x, t)}{\partial t}= \begin{cases}H^{*}\left(x, \frac{\partial J_{\epsilon}(x, t)}{\partial x}\right)+\epsilon \Delta J_{\epsilon}(x, t) & \text { for }\left\{x \in \mathbf{X}_{\mathbf{C}} \mid J_{\epsilon}(x, t)>0\right\} \\ \min \left\{0, H^{*}\left(x, \frac{\partial J_{\epsilon}(x, t)}{\partial x}\right)\right\}+\epsilon \Delta J_{\epsilon}(x, t) & \text { for }\left\{x \in \mathbf{X}_{\mathbf{C}} \mid J_{\epsilon}(x, t) \leq 0\right\}\end{cases}
$$

with boundary condition $J_{\epsilon}(x, 0)=l_{\epsilon}(x)$.

The level set methods of Osher and Sethian 8 ( 14 provides a comprehensive survey) are a set of computation schemes for propagating interfaces in which the speed of propagation is governed by a partial differential equation. These numerical techniques compute the viscosity solution to the Hamilton-Jacobi partial differential equation, ensuring that shocks are preserved. The methods have proved fruitful in many applications, including shape recovery problems in computer vision 15 , and plasma etching problems in micro chip fabrication 16 .

The key idea of the level set method is to embed the curve or surface to be evolved, for example the $n$-1-dimensional boundary of the reach set, as the zero level set of a function in $n$-dimensional space. The advantage of this formulation is that the $n$-dimensional function always remains a function as long as its speed of propagation is smooth, while the $n$-1-dimensional boundary may develop shocks or change topology under this evolution. The numerical methods of 14 choose the solution of 20 to be the one obtained from 20 as the viscosity coefficient $\epsilon$ vanishes. Below we present an outline of the method for a twodimensional example.

In order for the numerical scheme to closely approximate the gradient $\frac{\partial J^{*}(x, t)}{\partial x}$, especially at points of discontinuity, an appropriate approximation to the spatial derivative must be used. Consider an example in two dimensions, with $\mathbf{X}_{\mathbf{C}}$ discretized into a grid with spacing $\Delta x_{1}$ and $\Delta x_{2}$. As before we use $x=\left(x_{1}, x_{2}\right)$ to denote an element of $\mathbf{X}_{\mathbf{C}}$. The forward difference operator $D^{+x_{i}}$ is defined (for $x_{1}$, similarly for $x_{2}$ ) as:

$$
D^{+x_{1}} J(x, t)=\frac{J\left(\left(x_{1}+\Delta x_{1}, x_{2}\right), t\right)-J(x, t)}{\Delta x_{1}}
$$


The backward difference operator $D^{-x_{i}}$ is defined (for $x_{1}$, similarly for $x_{2}$ ) as

$$
D^{-x_{1}} J(x, t)=\frac{J(x, t)-J\left(\left(x_{1}-\Delta x_{1}, x_{2}\right), t\right)}{\Delta x_{1}}
$$

The central difference operator $D^{0 x_{i}}$ is defined (for $x_{1}$, similarly for $x_{2}$ ) as

$$
D^{0 x_{1}} J(x, t)=\frac{J\left(\left(x_{1}+\Delta x_{1}, x_{2}\right), t\right)-J\left(\left(x_{1}-\Delta x_{1}, x_{2}\right), t\right)}{2 \Delta x_{1}}
$$

At each grid point in $x$, the partial derivatives $\frac{\partial}{\partial x_{1}}$ and $\frac{\partial}{\partial x_{2}}$ may be approximated to first order using either the forward, backward, or central difference operators. The correct choice of operator depends on the direction of $f\left(x, u^{*}, d^{*}\right)$ (in our case it depends on $-f\left(x, u^{*}, d^{*}\right)$ since we compute backwards in time). If $-f\left(x, u^{*}, d^{*}\right)$ flows from left to right (from smaller to larger values of $x_{1}$ ), then then $D^{-x_{1}}$ should be used to approximate $\frac{\partial J(x, t)}{\partial x_{1}}$; and if $-f\left(x, u^{*}, d^{*}\right)$ flows from bottom to top (from smaller to larger values of $x_{2}$ ), then then $D^{-x_{2}}$ should be used to approximate $\frac{\partial}{\partial x_{2}}$ (and vice versa). Such an approximation is called an upwind scheme, since it uses information upwind of the direction that information propagates.

The algorithm for the two dimensional example proceeds as follows. Choose a domain of interest in $\mathbf{X}_{\mathbf{C}}$ and discretize the domain with a grid of spacing $\Delta x_{1}, \Delta x_{2}$. Let $x_{i j}$ represent the grid point $\left(i \Delta x_{1}, j \Delta x_{2}\right)$ and let $\tilde{J}\left(x_{i j}, t\right)$ represent the numerical approximation of $J\left(x_{i j}, t\right)$.

Set $t=0$ and compute the initial condition $\tilde{J}\left(x_{i j}, 0\right)=l\left(x_{i j}\right)$.

While for some $x_{i j}, \tilde{J}\left(x_{i j}, t\right) \neq \tilde{J}\left(x_{i j}, t-\Delta t\right)$ perform the following steps:

1. Compute $u^{*}\left(x_{i j}, D^{0 x_{1}} \tilde{J}\left(x_{i j}, t\right), D^{0 x_{2}} \tilde{J}\left(x_{i j}, t\right)\right)$ and $d^{*}\left(x_{i j}, D^{0 x_{1}} \tilde{J}\left(x_{i j}, t\right), D^{0 x_{2}} \tilde{J}\left(x_{i j}, t\right)\right)$.

2. Calculate $f\left(x_{i j}, u^{*}, d^{*}\right)$

3. If $\left(-f\left(x_{i j}, u^{*}, d^{*}\right)\right)$ flows from greater to lesser values of $x_{1}$, let $\frac{\partial}{\partial x_{1}}=D^{+x_{1}}$, otherwise let $\frac{\partial}{\partial x_{1}}=D^{-x_{1}}$.

4. If $\left(-f\left(x_{i j}, u^{*}, d^{*}\right)\right)$ flows from greater to lesser values of $x_{2}$, let $\frac{\partial}{\partial x_{2}}=D^{+x_{2}}$, otherwise let $\frac{\partial}{\partial x_{2}}=D^{-x_{2}}$.

5. Compute $\tilde{J}\left(x_{i j}, t-\Delta t\right)$ :

For $x_{i j}$ such that $\tilde{J}\left(x_{i j}, t\right)>0$,

$$
\tilde{J}\left(x_{i j}, t-\Delta t\right)=\tilde{J}\left(x_{i j}, t\right)+\Delta t \frac{\partial \tilde{J}\left(x_{i j}, t\right)}{\partial x} f\left(x_{i j}, u^{*}, d^{*}\right)
$$

For $x_{i j}$ such that $\tilde{J}\left(x_{i j}, t\right) \leq 0$,

$$
\tilde{J}\left(x_{i j}, t-\Delta t\right)=\left\{\begin{array}{c}
\tilde{J}\left(x_{i j}, t\right)+\Delta t \frac{\partial \tilde{J}\left(x_{i j}, t\right)}{\partial x} f\left(x_{i j}, u^{*}, d^{*}\right) \\
\text { if } \frac{\partial \tilde{J}\left(x_{i j}, t\right)}{\partial x} f\left(x_{i j}, u^{*}, d^{*}\right)<0 \\
\tilde{J}\left(x_{i j}, t\right) \text { otherwise }
\end{array}\right.
$$




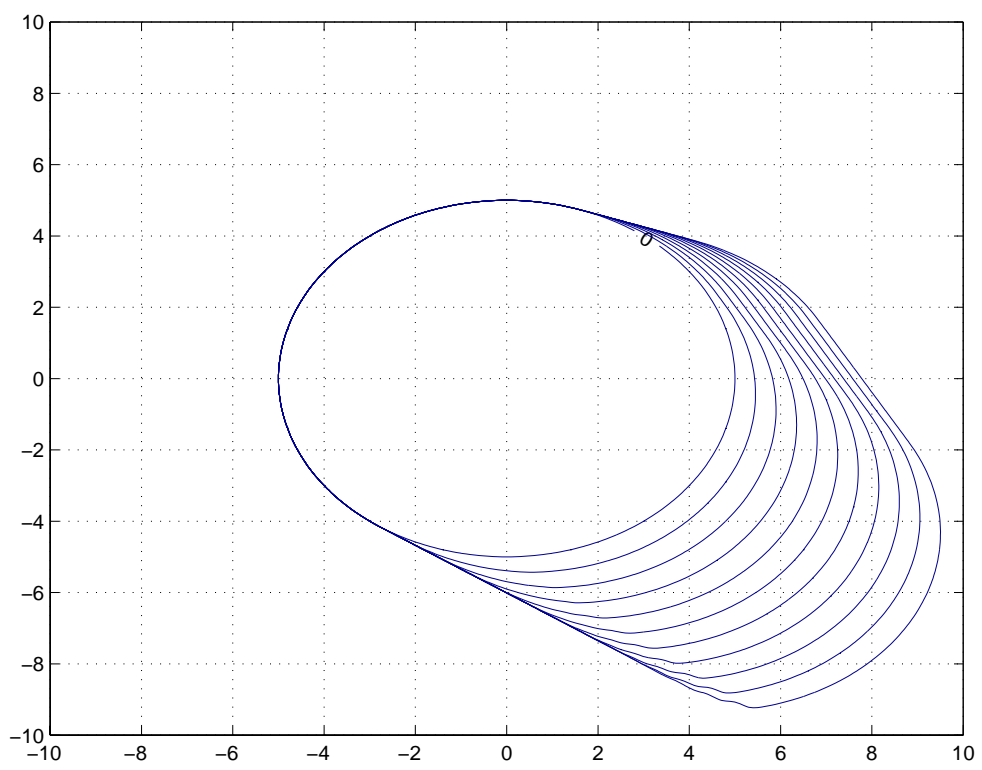

Fig. 2. $\left\{x \in \mathbf{X}_{\mathbf{C}} \mid J(x, t) \leq 0\right\}$ shown in the $\left(x_{r}, y_{r}\right)$-plane for $\left[\underline{v}_{1}, \bar{v}_{1}\right]=[2,4]$, $\left[\underline{v}_{2}, \bar{v}_{2}\right]=[1,5]$ and $\psi_{r}=2 \pi / 3$.

Figure $\boldsymbol{\lambda}$ displays the result of applying this algorithm to the two-aircraft example (presented in detail in Section $\underline{\varphi}$ ) with zero angular velocity and $\left[\underline{v}_{1}, \bar{v}_{1}\right]=$ $[2,4],\left[\underline{v}_{2}, \bar{v}_{2}\right]=[1,5]$ and $\psi_{r}=2 \pi / 3$.

The above discussion presents the very basic idea of level set methods; for special forms of the Hamilton-Jacobi equation, extremely efficient variants of this method exist 14 . In particular, the narrow band and fast marching methods speed up the algorithm by confining the computation to a narrow band around the evolving front.

\subsection{Other Methods}

A number of other techniques have been proposed for numerically approximating the set of states reachable by a hybrid system, primarily for the purpose of verifying safety properties. We review some of them below.

Polyhedral Approximations Consider a hybrid automaton with no control inputs. For each valuation of the discrete state, the continuous dynamics of such an automaton can be captured by a differential inclusion:

$$
\dot{x} \in g(x)=\{f(x, d) \mid d \in \mathbf{D}\}
$$


One technique for numerically approximating the set of states that can be reached by such an automaton is to partition the continuous state space into polyhedral regions (e.g. rectangles) and then to conservatively approximate $g(x)$ in each region by a constant inclusion of the form:

$$
\dot{x} \in\left[g_{\min }, g_{\max }\right]
$$

The computation of the reach set for the approximate system can then be carried out exactly using tools developed for linear hybrid automata. In $\mathrm{I7}$ it is shown that the error in approximating the set of states reachable by the true system in a bounded time interval can be made arbitrarily small by approximating the differential inclusion arbitrarily closely. An advantage of this method is that the class of constant inclusions used to approximate the differential inclusion is known to be decidable, thus one can guarantee that the reachable set of the approximate system can be computed in a finite number of steps. However, the amount of preprocessing required to initially approximate the dynamics may be formidable, especially if the time horizon over which the reach set needs to be calculated is large.

A related approach is that of 18 and 19 . Here the approximation of the dynamics is not carried out a-priori over the entire space, but only locally around the boundary of the reachable set as the latter propagates in time. This could potentially lead to substantial computational savings. The class of sets generated by these techniques is again polyhedral, but no a-priori bounds are given on the accuracy of the approximation.

Approximating non-smooth sets with smooth sets We have shown that the reach set at any time $t \in(-\infty, 0]$ may have a non-smooth boundary due to switches in $\left(u^{*}, d^{*}\right)$, non-smooth initial data, or the formation of shocks. The level set method scheme propagates these discontinuities, yet its implementation may require a very small time step to do this accurately. In 7 we present a method for over-approximating such non-smooth sets with sets for which the boundary is continuously differentiable. Suppose that there exist differentiable functions $l_{G}^{i}, i=1, \ldots, k$ such that for $G$ a closed subset of $\mathbf{X}_{\mathbf{C}}$ :

$$
G=\left\{x \in \mathbf{X}_{\mathbf{C}} \mid \forall i \in\{i=1, \ldots, k\}, l_{G}^{i}(x) \leq 0\right\}
$$

Following 2021 we define two smooth functions:

$$
\begin{aligned}
& G^{\epsilon}(x)=\epsilon \ln \left[\sum_{i=1}^{k} e^{l_{G}^{i}(x) / \epsilon}\right] \\
& G_{\epsilon}(x)=G^{\epsilon}(x)-\epsilon \ln k
\end{aligned}
$$

Define:

$$
\begin{aligned}
& G_{\epsilon}=\left\{x \in \mathbf{X}_{\mathbf{C}} \mid G_{\epsilon}(x) \leq 0\right\} \\
& G^{\epsilon}=\left\{x \in \mathbf{X}_{\mathbf{C}} \mid G^{\epsilon}(x) \leq 0\right\}
\end{aligned}
$$


One can show that $G_{\epsilon} \subseteq G \subseteq G^{\epsilon}$ anc ${ }^{2} \lim _{\epsilon \rightarrow 0} G_{\epsilon}=\lim _{\epsilon \rightarrow 0} G^{\epsilon}=G$. By applying Algorithm 9 to smooth inner and outer approximations of the set $G$, one can calculate smooth inner and outer approximations to the reach set.

A similar idea is to use ellipsoids as inner and outer approximations to the reach set 222.3 .2 .3 presents efficient algorithms for calculating both the minimum volume ellipsoid containing given points, and the maximum volume ellipsoid in a polyhedron, using matrix determinant maximization subject to linear matrix inequality constraints.

\section{Two-Aircraft Conflict Resolution}

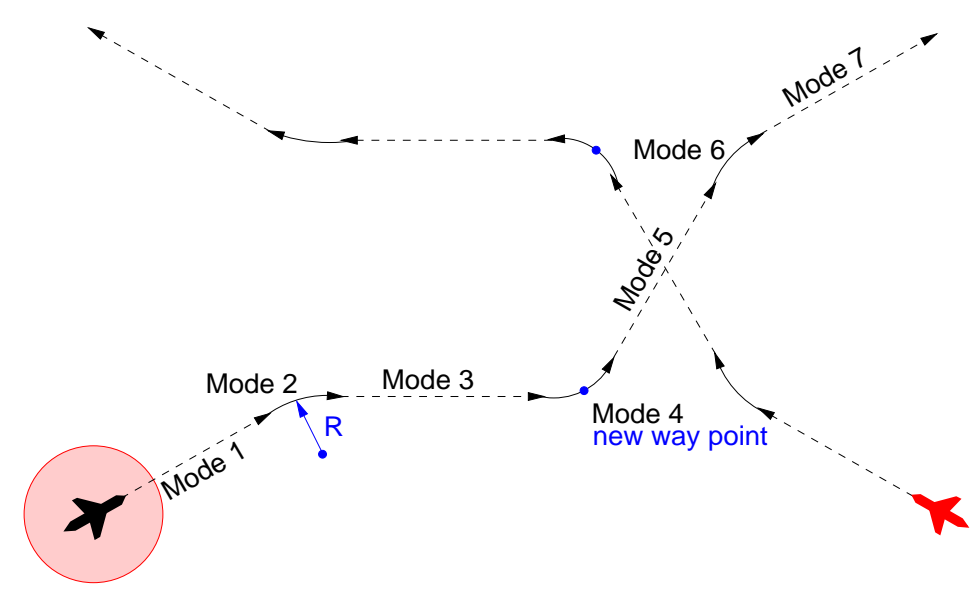

Fig. 3. Two aircraft in seven modes of operation: in modes $1,3,5$, and 7 the aircraft follow a straight course and in modes 2,4 , and 6 the aircraft follow arcs of circles. The initial relative heading is preserved throughout.

Consider two aircraft flying in collision course on the same horizontal plane (Figure 3). To avoid the collision the aircraft go through a coordinated avoidance maneuver: when they come within a certain distance of each other, they both start to turn to the right, following a trajectory which is a sequence of arcs of circles of fixed radii, and straight lines (trimmed flight segments). We assume that aircraft 1 initiates the avoidance maneuver and that the aircraft communicate and switch modes simultaneously. We also assume that the angles of the avoid maneuver are fixed, so that the straight path of mode 3 is at a $-45^{\circ}$ angle to the straight path of mode 1 , and that of mode 5 is at a $45^{\circ}$ to that of mode 1 . Also, the radius of each arc is fixed at a pre-specified value, and the lengths of the segments in modes 3 and 5 are equal to each other, but unspecified. Given

$\overline{{ }^{2} \text { Considering }}$ appropriate topologies for $2^{\mathbf{x}_{\mathbf{C}}}$. 
some uncertainty in the actions of the aircraft, we would like to generate the relative distance between aircraft at which the aircraft may switch safely from mode 1 to mode 2, and the minimum lengths of the segments in modes 3 and 5 , to ensure that a 5 nautical mile separation is maintained.

The system can be modeled by a hybrid automaton with seven discrete states $\left(\mathbf{X}_{\mathbf{D}}=\{\right.$ straight1, arc1, straight2, arc2, straight3, arc3, straight 4$\left.\}\right)$ and four continuous states, the relative position, $\left(x_{r}, y_{r}\right)$, and heading, $\psi_{r}$, of the two aircraft, and a clock variable, $z$, to keep track of how long the aircraft have stayed in each mode. Overall, $\mathbf{X}_{\mathbf{C}}=\mathbb{R}^{2} \times[0,2 \pi] \times \mathbb{R}$. A discrete control input $\sigma \in \mathbf{U}_{\mathbf{D}}=\{0,1\}$ can be used to initiate the maneuver. There is also a continuous control input, the groundspeed of aircraft $1, v_{1} \in \mathbf{U}_{\mathbf{C}}=\left[\underline{v_{1}}, \overline{v_{1}}\right]$ and a continuous disturbance input, the groundspeed of aircraft $2, v_{2} \in \overline{\mathbf{D}}=\left[\underline{v_{2}}, \overline{v_{2}}\right]$. The speed of aircraft 2 is treated as a disturbance because we assume that aircraft 1 can estimate it only approximately. The dynamics are shown pictorially in Figure $1 !$ in the usual location invariant, transition guard and transition reset relation convention. The unsafe set $G$ is given by:

$$
G=\mathbf{X}_{\mathbf{D}} \times\left\{\left(x_{r}, y_{r}, \psi_{r}, z\right) \in \mathbf{X}_{\mathbf{C}} \mid x_{r}^{2}+y_{r}^{2} \leq 5^{2}\right\}
$$

To simplify the calculation we assume that the speed of both aircraft remains

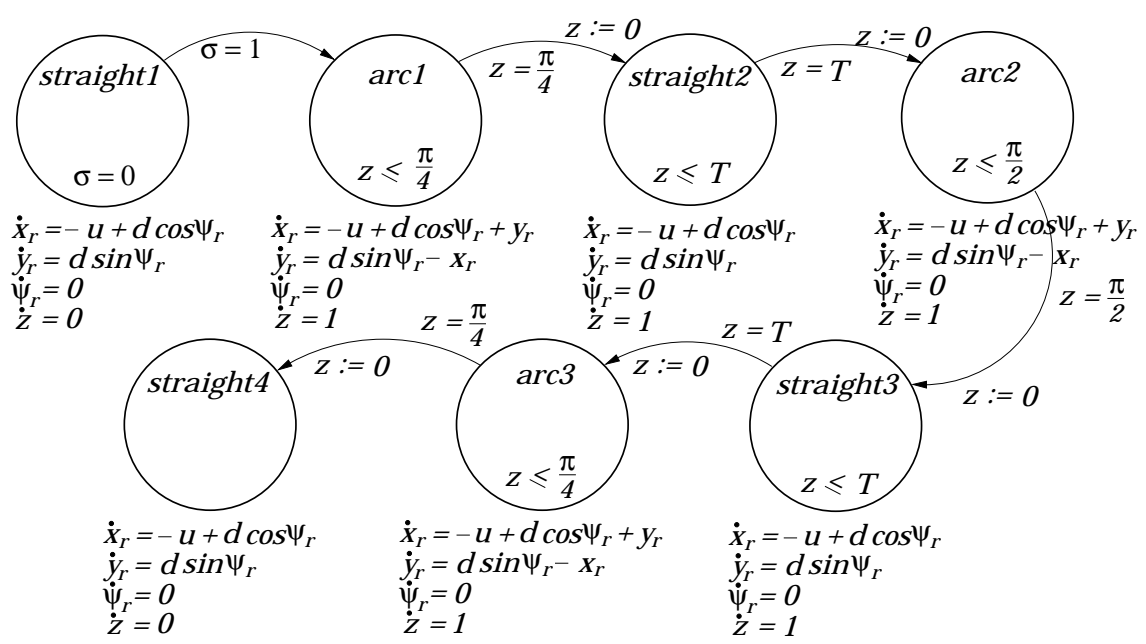

Fig. 4. Hybrid automaton model for the conflict resolution maneuver.

constant during the circular parts of the maneuver, but can take on any allowable value in the straight parts. In other words, $\phi(x)=\mathbf{U}_{\mathbf{D}} \times\left\{\left(\hat{v}_{1}, \hat{v}_{2}\right)\right\}$ if $\left.x\right|_{X_{D}} \in$ $\{\operatorname{arc1}, \operatorname{arc2}, \operatorname{arc} 3\}$ and $\phi(x)=\mathbf{U}_{\mathbf{D}} \times \mathbf{U}_{\mathbf{C}} \times \mathbf{D}$ otherwise.

Our goal is to compute the relative distance at which the maneuver must start, the length of the straight legs straight2 and straight3, as well as the groundspeed $v_{1}^{*}$ along those legs, to ensure safety. The unsafe sets computed 

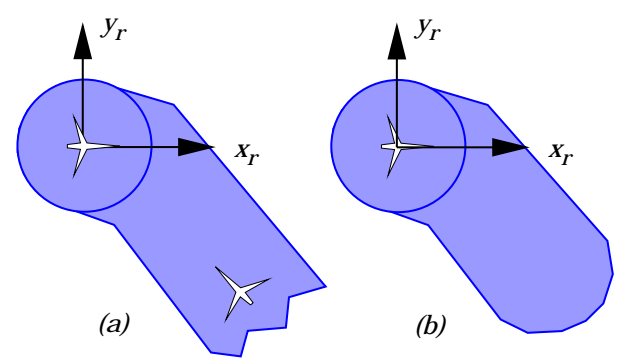

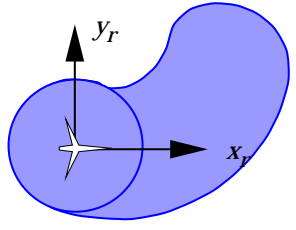

(c)

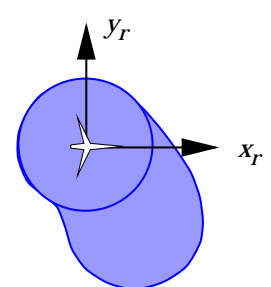

(d)

Fig. 5. $J_{G_{i}}(x) \leq 0$ for (a) Modes 1 and $7(i=1,7), \omega_{1}=\omega_{2}=0$ and $\left[\underline{v}_{1}, \bar{v}_{1}\right]=$ $[2,4],\left[\underline{v}_{2}, \bar{v}_{2}\right]=[1,5]$ (the jagged edge means the set extends infinitely); (b) Modes 3 and $5(i=3,5), \omega_{1}=\omega_{2}=0$ and $\left[\underline{v}_{1}, \bar{v}_{1}\right]=[2,4],\left[\underline{v}_{2}, \bar{v}_{2}\right]=[1,5] ;$ (c) Mode $4(i=4), \omega_{1}=\omega_{2}=1$ and $v_{1}=v_{2}=5$; and (d) Modes 2 and $6(i=2,6)$, $\omega_{1}=\omega_{2}=-1$ and $v_{1}=v_{2}=5$. In all cases, $\psi_{r}=2 \pi / 3$.

by our algorithm for each one of the discrete modes are shown in Figure $\mathbf{5}$ for $i=1, \ldots, 7$. In the straight modes, the sets are calculated using $\left(v_{1}^{*}, v_{2}^{*}\right)$ of Section 1 (and thus show a close resemblance to the set in Figure $\boldsymbol{\nu}$. The corners in the set for the straight modes $(1,3,5$ and 7$)$ indicate discontinuities in the optimal controls $v_{1}^{*}$ and $v_{2}^{*}$. Figure 6 displays the fixed point $W^{*}=W^{-7}$ for the initial mode straight1. The controller that switches between the modes is also illustrated in Figure 6 The time spent in the straight legs of the maneuver $T$, may be chosen to minimize the deviation from the original route, while ensuring that $\left(W^{*}\right)^{c}$ is small.

\section{Concluding Remarks}

A common concern for all approximations for the computation of the set of reachable states is that, for safety properties, one typically would like a conservative over approximation. This will ensure that if the approximate set of reachable states satisfies the property the exact set will also satisfy it. This requirement is built into most of the approximation techniques discussed in Section 4 It is not as easy to satisfy with the level set method, however. One needs to keep accurate bounds of the numerical errors and grow the final estimate of the reach set appropriately.

An additional issue one needs to consider in the context of controller synthesis is the controlled invariance of the approximation. This issue has not to our knowledge been addressed by any of the methods proposed in the literature (since they are primarily concerned with verification). One would like to ensure that the numerical estimate of the reach set (for the case of the level set method, this could be some interpolation between the collection of discrete points produced by the algorithm) is controlled invariant. If this is indeed the case, one would also like to obtain a controller that renders the approximation invariant. 


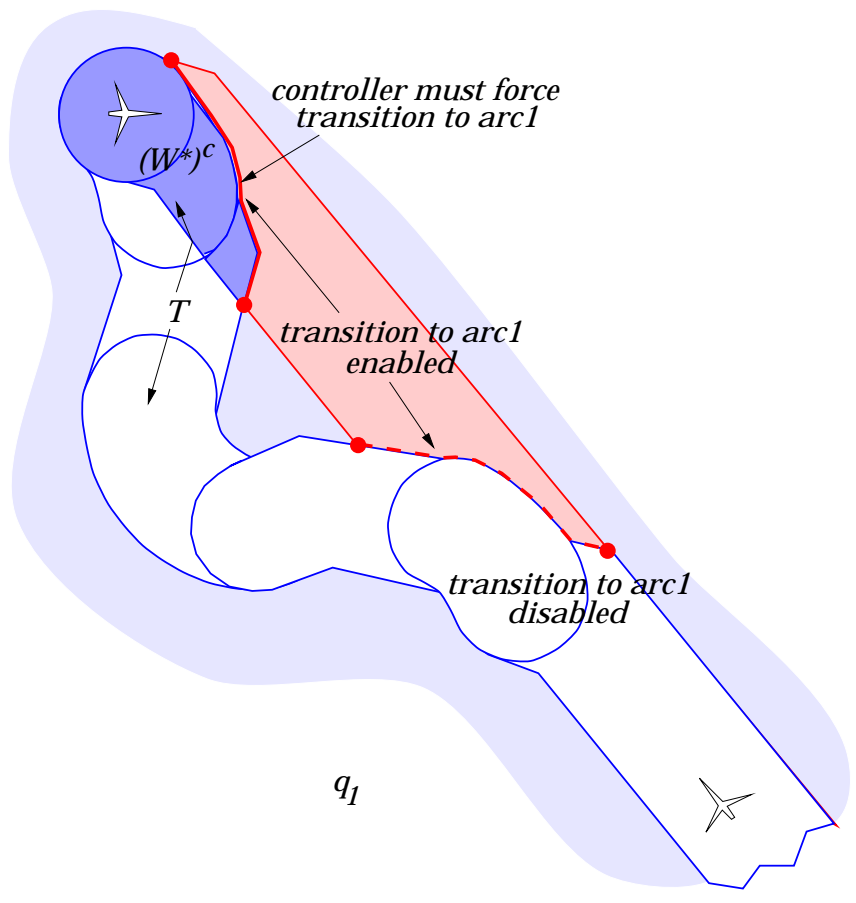

Fig. 6. $\left(W^{*}\right)^{c}=\left(W^{-7}\right)^{c}$ in straight1.

\section{References}

1. W. Thomas, "On the synthesis of strategies in infinite games", in Proceedings of STACS 95, Volume 900 of LNCS, Ernst W. Mayr and Claude Puech, Eds., pp. 1-13. Springer Verlag, Munich, 1995.

2. T. Başar and G. J. Olsder, Dynamic Non-cooperative Game Theory, Academic Press, second edition, 1995.

3. O. Maler, A. Pnueli, and J. Sifakis, "On the synthesis of discrete controllers for timed systems", in STACS 95: Theoretical Aspects of Computer Science, Ernst W. Mayr and Claude Puech, Eds., Lecture Notes in Computer Science 900, pp. 229242. Springer Verlag, Munich, 1995.

4. H. Wong-Toi, "The synthesis of controllers for linear hybrid automata", in Proceedings of the IEEE Conference on Decision and Control, San Diego, CA, 1997.

5. J. Lygeros, C. Tomlin, and S. Sastry, "Controllers for reachability specifications for hybrid systems", Automatica, vol. 35, no. 3, 1999, To appear.

6. C. Tomlin, J. Lygeros, and S. Sastry, "Synthesizing controllers for nonlinear hybrid systems", in Hybrid Systems: Computation and Control, T. Henzinger and S. Sastry, Eds., Lecture Notes in Computer Science 1386, pp. 360-373. Springer Verlag, New York, 1998.

7. J. Lygeros, C. Tomlin, and S. Sastry, "On controller synthesis for nonlinear hybrid systems", in Proceedings of the IEEE Conference on Decision and Control, Tampa, FL, 1998, pp. 2101-2106. 
8. S. Osher and J. A. Sethian, "Fronts propagating with curvature-dependent speed: Algorithms based on Hamilton-Jacobi formulations", Journal of Computational Physics, vol. 79, pp. 12-49, 1988.

9. Claire J. Tomlin, Hybrid Control of Air Traffic Management Systems, PhD thesis, Department of Electrical Engineering, University of California, Berkeley, 1998.

10. Michael S. Branicky and Sanjoy K. Mitter, "Algorithms for optimal hybrid control", in Proceedings of the IEEE Conference on Decision and Control, 1995, pp. 2661-2666.

11. Adam L. Schwartz, Theory and Implementation of Numerical Methods Based on Runge-Kutta Integration for Solving Optimal Control Problems, $\mathrm{PhD}$ thesis, Department of Electrical Engineering, University of California, Berkeley, 1996.

12. M. G. Crandall and P.-L. Lions, "Viscosity solutions of Hamilton-Jacobi equations", Transactions of the American Mathematical Society, vol. 277, no. 1, pp. 1-42, 1983.

13. M. G. Crandall, L. C. Evans, and P.-L. Lions, "Some properties of viscosity solutions of Hamilton-Jacobi equations", Transactions of the American Mathematical Society, vol. 282, no. 2, pp. 487-502, 1984.

14. J. A. Sethian, Level Set Methods: Evolving Interfaces in Geometry, Fluid Mechanics, Computer Vision, and Materials Science, Cambridge University Press, New York, 1996.

15. R. Malladi, J. A. Sethian, and B. C. Vemuri, "Shape modeling with front propagation: A level set approach", IEEE Transactions on Pattern Analysis and Machine Intelligence, vol. 17, no. 2, pp. 158-175, 1995.

16. J. M. Berg, A. Yezzi, and A. R. Tannenbaum, "Phase transitions, curve evolution, and the control of semiconductor manufacturing processes", in Proceedings of the IEEE Conference on Decision and Control, Kobe, 1996, pp. 3376-3381.

17. A. Puri, P. Varaiya, and V. Borkar, " $\epsilon$-approximation of differential inclusions", in Proceedings of the IEEE Conference on Decision and Control, New Orleans, LA, 1995, pp. 2892-2897.

18. T. Dang and O. Maler, "Reachability analysis via face lifting", in Hybrid Systems: Computation and Control, S. Sastry and T.A. Henzinger, Eds., number 1386 in LNCS, pp. 96-109. Springer Verlag, 1998.

19. M.R. Greenstreet and I. Mitchell, "Integrating projections", in Hybrid Systems: Computation and Control, S. Sastry and T.A. Henzinger, Eds., number 1386 in LNCS, pp. 159-174. Springer Verlag, 1998.

20. D. P. Bertsekas, Constraint Optimization and Lagrange Multiplier Methods, Academic Press, New York, 1982.

21. E. Polak, Optimization: Algorithms and Consistent Approximations, Springer Verlag, New York, 1997.

22. A. B. Kurzhanski and I. Valyi, Ellipsoidal calculus for estimation and control, Birkhauser, Boston, 1997.

23. L. Vandenberghe, S. Boyd, and S.-P. Wu, "Determinant maximization with linear matrix inequality constraints", SIAM Journal on Matrix Analysis and Applications, vol. 19, no. 2, pp. 499-533, 1998. 